\title{
Depression-the hidden symptom in advanced cancer
}

Psychiatric disorders are more prevalent in patients with cancer than in the general population. ${ }^{1,2}$ About $30 \%$ of such patients will be experiencing an 'adjustment reaction' and $20 \%$ will qualify for a formal psychiatric diagnosis, most commonly depression. ${ }^{3}$ For a quarter of all patients admitted to a palliative care unit, depression will be a significant symptom. ${ }^{4}$ Even in the general population the prevalence of depression is $6-10 \%$, and those with a preexisting psychiatric disorder will be at special risk. ${ }^{5}$

More than 40 years have passed since Hinton drew attention to the issue of mental distress in the dying 6 yet surveys still point to much unrecognized suffering; ${ }^{7}$ as much as $80 \%$ of the psychological and psychiatric morbidity of patients with cancer goes unrecognized and untreated. One reason is that many patients choose not to disclose their symptoms - perhaps because they feel it is a waste of the professional's time or because they think they are in some way blameworthy. ${ }^{8}$ But another factor may be that medical and nursing staff lack confidence in diagnosing depression in these circumstances. ${ }^{9}$ In this article I review the methods that can help in detection of depression and the options for intervention when it is identified.

\section{HOW IS DEPRESSION DIAGNOSED?}

There are no universally accepted criteria for diagnosing depression in the terminally ill patient. In the physically healthy population, depression is diagnosed if patients have a persistent low mood and at least four of the following symptoms present most of the day for the preceding two weeks:

1 Diminished interest or pleasure in all or almost all activities

2 Psychomotor retardation or agitation

3 Feelings of worthlessness or excessive and inappropriate guilt

4 Diminished ability to concentrate and think

5 Recurrent thoughts of death and suicide

6 Fatigue and loss of energy

7 Significant weight loss or gain

8 Insomnia or hypersomnia.

University of Liverpool Medical School, Department of Primary Care, Harrison Hughes Building, Brownlow Hill, Liverpool L69 3GB, UK

E-mail: mlw@liv.ac.uk
In patients with advanced cancer, symptoms 6-8 are almost universal and there was much controversy over whether they should be included and, if so, their importance in the diagnosis of depression in such patients. Some workers reported that feelings of worthlessness, helplessness and hopelessness, feelings of excessive and inappropriate guilt and thoughts of self-harm were particularly discriminating; ${ }^{10,11}$ indeed, when somatic symptoms were omitted from the criteria, the point prevalence of major depression dropped from $42 \%$ to $24 \% .{ }^{11}$ Discussing the complex matter of which symptoms are attributable to the cancer and which to depression, Endicott ${ }^{12}$ proposed modified criteria for depression in which alternatives were substituted for the somatic symptoms - for instance, instead of 'poor appetite', 'fearfulness or depressed appearance in body or face'. Endicott also stressed the importance of asking patients with cancer about suicidal ideation. When the research diagnostic criteria were compared with Endicott's criteria, it was found that small differences in the application of symptom severity thresholds could cause large differences in prevalence rates for depression. ${ }^{13}$ The inclusion of somatic symptoms only inflated the rates of diagnosis when these symptoms were used with a 'low threshold' approach.

Whilst some patients require expert psychiatric assessment, many can be adequately assessed by a doctor or nurse who has acquired the basic skills. Ideally there should be an integrated referral system that offers ready access to a mental health professional when needed. At present few hospices have psychiatric input of this sort. ${ }^{14}$

\section{HOW CAN DEPRESSION BE ASSESSED?}

A particular difficulty in assessment is that patients underestimate their own distress, and the opinion of a close friend or relative tends to be unhelpful, reflecting his or her own distress rather than that of the patient. ${ }^{15}$ Hoeper et al. ${ }^{16}$ found that doctors were influenced by previous psychiatric history when diagnosing depression, and suggested that the process of screening for mental illness could itself be therapeutic, in prompting the patient to disclose more symptoms.

One method of assessing depression is to use rating scales. Below I discuss the most frequently used scales and why those used in clinical trials may be unsatisfactory in palliative care. 
Table 1 The efficacy of the HAD scale in identifying depression according to ICD 10 criteria using the combined anxiety and depression subscales and Present State Examination interview

\begin{tabular}{|c|c|c|c|c|c|c|c|c|c|}
\hline & \multicolumn{9}{|c|}{ Threshold scores } \\
\hline & 14 & 15 & 16 & 17 & 18 & 19 & 20 & 21 & 22 \\
\hline Sensitivity (\%) & 77 & 77 & 72 & 72 & 68 & 68 & 54 & 54 & 45 \\
\hline Specificity (\%) & 37 & 44 & 50 & 56 & 63 & 67 & 70 & 74 & 83 \\
\hline PPV (\%) & 25 & 28 & 29 & 32 & 34 & 36 & 34 & 37 & 43 \\
\hline NPV (\%) & 85 & 87 & 86 & 86 & 87 & 88 & 84 & 85 & 84 \\
\hline
\end{tabular}

$\mathrm{HAD}=$ Hospital Anxiety and Depression Scale; ICD=International Classification of Diseases; PPV, NPV=positive and negative predictive values

\section{The Hospital Anxiety and Depression Scale}

The Hospital Anxiety and Depression Scale (HAD) is a fourteen-item scale devised in 1983 by Zigmond and Snaith for use with medical patients. ${ }^{17} \mathrm{~A}$ main characteristic is the exclusion of symptoms that may have both an emotional and a physical aetiology - e.g. dizziness or sleep disturbanceand it is a widely used depression rating tool in cancer and palliative care. The depression scale is based on anhedonia - the complete loss of enjoyment or 'exclusion from the pleasure dome'. ${ }^{18}$ The authors of the scale stated that this symptom would indicate which patients might respond to antidepressant medication. ${ }^{19}$ The General Health Questionnaire (GHQ), the Rotterdam Symptom Checklist (RSCL) and the HAD scale together with the Psychiatric Assessment Schedule were assessed by Ibbotson et al. ${ }^{20}$ in a prospective study of 513 patients with cancer. The HAD scale performed best in patients who were disease-free or receiving treatment. Anhedonia, which is measured by five of the seven items on the HAD depression subscale, is a very common feature in patients and cannot be relied upon alone to differentiate depressive illness from other mental illness. ${ }^{21}$ Le Fevre, ${ }^{22}$ comparing the HAD and the GHQ, recommended summing of the scores on anxiety and depression subscales rather than use of the depression subscale alone. A combined cut-off score of 20 achieves a sensitivity of 0.77 and specificity of 0.85 and a positive predictive value of 0.48 . A further study ${ }^{23}$ in 100 patients receiving palliative care with an estimated prognosis of six months or less showed that, when used as a screening tool, the depression and anxiety scales of the HAD were of low efficacy when used singly. The optimum threshold was at a combined cut-off of 19 , which had a sensitivity of 0.68 , a specificity of 0.67 and a positive predictive value of 0.36 (Table 1).

\section{The Zung Self-rating Depression Scale}

The Zung Self-rating Scale ${ }^{24}$ is a twenty-item self-report measure of the symptoms of depression in which patients
Table 2 The Edinburgh Depression Scale with use of Present State Examination interview to identify cases of depression according to ICD 10 criteria

\begin{tabular}{|c|c|c|c|c|c|c|c|c|c|}
\hline & \multicolumn{9}{|c|}{ Threshold scores } \\
\hline & 8 & 9 & 10 & 11 & 12 & 13 & 14 & 15 & 16 \\
\hline Sensitivity (\%) & 100 & 100 & 100 & 95 & 86 & 81 & 68 & 50 & 50 \\
\hline Specificity (\%) & 31 & 37 & 52 & 61 & 66 & 79 & 85 & 89 & 94 \\
\hline PPV (\%) & 29 & 31 & 36 & 42 & 42 & 53 & 55 & 58 & 73 \\
\hline NPV (\%) & 100 & 100 & 100 & 97 & 94 & 94 & 91 & 86 & 86 \\
\hline
\end{tabular}

ICD=International Classification of Diseases; PPV, NPV=positive and negative predictive values

rate their symptoms according to how they have felt in the previous week. Scores are categorized into four different levels reflecting the presence or absence of depressive symptoms. As with other screening tools the aim is not to make a diagnosis but to identify patients with clinical symptoms suggestive of depressive illness. The Zung scale and the briefer eleven-item Zung scale (which excludes nine items relating to somatic symptoms) has been found useful in cancer patients, predominantly those with stable disease. ${ }^{25,26}$

\section{The Edinburgh Depression Scale}

The Edinburgh scale was devised by Cox et al. ${ }^{27,28}$ as a screening tool for postnatal depression. With its ten items selected to exclude the somatic symptoms of depression, it was devised to be administered by healthcare workers with no specialist knowledge of psychiatry. The original scale was validated in 84 mothers in the postnatal period and found to have a sensitivity of 0.86 and specificity of 0.78 (with cutoff threshold 12/13). The authors believed it could be used for other populations and the questions concerning guilt, helplessness/hopelessness, low mood and thoughts of selfharm seemed particularly important diagnostic symptoms of depression in the terminally ill. The Edinburgh Scale was therefore assessed against the Present State Examination for depression according to International Classification of Diseases (10th edition) criteria in 100 inpatients with metastatic cancer who were receiving palliative care. In this study a cut-off threshold of 13 gave optimum sensitivity 0.81 and specificity 0.79 , with positive predictive value 0.53 (Table 2). ${ }^{29}$

\section{Visual analogue scales and simple questions ('Are you depressed?')}

Answers to the simple question 'are you depressed?' can be informative. ${ }^{30,31}$ Chochinov in the USA assessed 197 palliative care inpatients using four screening tools together with a diagnostic interview for depression according to 
Research Diagnostic Criteria. The diagnostic interview was adapted from the Schedule for Affective Disorders and Schizophrenia (SADS). The question 'are you depressed?' (taken from the full SADS interview) correctly identified the eventual diagnostic outcome of every patient (sensitivity, specificity and positive predictive value all 1.0). Addition of a second question about loss of interest reduced the specificity $(0.98)$ and positive predictive value (0.86) though not the sensitivity. Work in the UK has yielded less favourable results. ${ }^{32}$ When assessed against a clinical psychiatric interview, the question 'are you depressed?' had sensitivity and specificity less than 0.6 . A possible reason is that, in the original study, the question was included also in the validating interview; moreover, the responses will depend greatly on interviewers' and patients' understanding of the concept of depression.

\section{Thoughts of self-harm}

Although by no means diagnostic, thoughts of self-harm are important indicators of depression in the terminally ill patient. In any form of psychiatric interview or screening in such patients they should be inquired about. An overwhelming feeling of helplessness and hopelessness is an indicator of suicidal risk ${ }^{33}$ and such feelings are commonly reported also by patients who are depressed. Brown et al..$^{34}$ and Chochinov et al. ${ }^{35}$ found that, among terminally ill patients, suicidal thoughts and desire for death were almost entirely confined to patients with a psychiatric disorder. Tiernan et al. ${ }^{36}$ examined the question by exploring the relations between desire for early death, depressive symptoms and antidepressant prescribing in 142 patients. They used the HAD scale and four additional questions - 'I go to sleep hoping that I won't wake up'; 'I think of ending my life but I would not do it'; 'I would end my life if I had a chance'; and 'I wish that doctors would do something to end my life'. 120 patients stated they had never desired an early death and only 2 patients had a strong wish for death. Depressive symptoms were frequently identified by the HAD scale, but the components of the HAD scale may mean that the true levels of depression were underestimated. ${ }^{23}$ The desire for early death correlated strongly with the depression scores - as found also by others. Tiernan found that only $12 \%$ of patients were receiving antidepressant medication and judged that antidepressants were underutilized in this population. ${ }^{38}$

What of actual suicide in patients with cancer? Farebarrow, ${ }^{39}$ reporting on 16 severely incapacitated patients who committed suicide, concluded that intention rather than physical strength is the important variable. Advanced physical disability was also present in more than two-thirds of the 88 cancer suicides studied by Bolund. ${ }^{40,41}$
35 of these patients had developed a mental illness after the cancer was diagnosed, the most frequent conditions being depression and what Bolund terms a 'reaction to illness' (presumably an adjustment reaction).

\section{THERAPEUTIC OPTIONS FOR DEPRESSION}

\section{Non-pharmacological therapy}

Patients with depression require psychotropic medication but wellbeing and recovery are also promoted by psychological support. Over the past 10 years there has been an enormous increase in the availability and uptake of counselling, but there is little evidence of benefit from counselling in depression. There is only one report of a significant improvement in quality of life within three months of counselling, ${ }^{42}$ and in this study the counsellors were very closely engaged, visiting the patient several times a week.

Psychotherapy is designed to achieve lasting change and in the psychiatric setting is often used for patients with deep-seated psychopathology. Spiegel ${ }^{43}$ identified four basic components of psychotherapy when used in patients with cancer-namely, social support, especially when group psychotherapy is used; emotional expression; cognitive restructuring; and coping skills training. There are indications that cancer patients who become depressed have a distinct pattern of coping skills. ${ }^{44}$

Adjuvant psychological therapy has been found beneficial in patients with cancer. The theoretical framework is founded on a premise that cancer-related psychological distress stems from the personal meaning of the disease to the patient and the patient's own coping skills. ${ }^{45}$ Patients are seen for six one-hour sessions, the main aims being to induce a fighting spirit, to encourage expression of emotions, to challenge negative behaviour and to look at cognitive techniques for countering negative behaviour. In a further study ${ }^{46}$ this strategy outperformed counselling in terms of sustained fighting spirit, coping with cancer, anxiety and self-defined problems. This latter study included patients at various stages of cancer including advanced metastatic disease. Clearly psychological support is important for all patients with cancer, and the relative merits of counselling and focused psychotherapy are still debated. In practice, the choice is limited by the extreme shortage of psychologists in the UK; indeed, the popularity of counselling within cancer and palliative care services may reflect the ease of access to counsellors rather than evidence that counselling is of major benefit. A study in primary care has suggested that, although counselling can be of value in depression, ${ }^{47}$ antidepressants act faster and therefore may be more appropriate for patients with short life expectancy. 


\section{Psychotropic medication}

In patients with cancer the principles of treatment with antidepressant medication are the same as in any other patient. ${ }^{48}$ The underprescribing of antidepressants probably stems from misconceptions - for example, the notions that medication is pointless when patients have an obvious reason for being depressed, that psychological treatments are better or that drug treatment is incompatible with psychological treatment.

A patient with cancer starting on an antidepressant has a probability of responding similar to that of depressed patients in the general population. In a study of 156 patients with cancer referred to a liaison psychiatry service $^{49}$ antidepressants were prescribed to two-thirds of the patients and $80 \%$ showed a good clinical response with few side-effects. Tricyclic antidepressants are frequently used in palliative care for the treatment of neuropathic pain, but the doses for this purpose are much lower than those required for an antidepressant effect. With full dosage their anticholinergic side-effects are commonly troublesome in palliative care patients treated for depression. There is less evidence for the use of selective serotonin reuptake inhibitors in treatment of neuropathic pain, though sertraline and paroxetine have been reported to relieve the pain of diabetic neuropathy. ${ }^{50,51}$ The selective serotonin reuptake inhibitors lack the anticholinergic cardiac and sedative effects of the tricyclic drugs and are safer in overdose but may cause nausea, diarrhoea, anxiety and insomnia. Few studies have been done in depressed patients with advanced cancer. ${ }^{52}$ All antidepressants take about three weeks to work but side-effects tend to begin in the first few days and hamper adherence to treatment. One of the newer antidepressants mirtazapine seems clinically to have a good side-effect profile when used for patients with advanced cancer. ${ }^{53}$ The choice of antidepressant is therefore governed by a decision on which drug is best suited to the individual patient. In a large community study selective serotonin reuptake inhibitors were better tolerated and more likely to be continued than tricyclic antidepressants. ${ }^{54}$

\section{Psychostimulants}

Psychostimulants such as dexamphetamine increase the sense of well-being and improve mood. They work by promoting the release of biogenic amines and must be taken early in the day to avoid insomnia. The dose is normally maintained for seven to ten days and is then slowly reduced. They are generally safe, but care needs to be taken in patients with multiple organ failure. Psychostimulants are used frequently in the United States ${ }^{55,56}$ but seldom in the UK or continental Europe. ${ }^{57}$

\section{CONCLUSIONS}

Adequate recognition and treatment of depression in patients with cancer can enhance quality of life and help patients and families make best use of their remaining time together. ${ }^{58,59}$ Since patients are commonly reluctant to describe their depressive symptoms, staff need to know how depression can be recognized.

Screening tools have a role, if used judiciously. Assessment of depression requires sensitive questioning about the patient's perception of his or her mood together with inquiry on areas such as guilt, hopelessness, helplessness and suicidal thoughts.

\section{REFERENCES}

1 Block S. Assessing and managing depression in the terminally ill patient. Ann Intern Med 2000;132:209-18

2 Brietbart W, Bruera E, Chochinov H, Lynch M. Neuropsychiatric syndromes and psychological symptoms in patients with advanced cancer. J Pain Symptom Management 1995;10:131-41

3 Hughes J. Depressive illness and lung cancer. II. Follow-up of inoperable patients. Eur J Surg Oncol 1985;11:21-4

4 Barraclough J. Cancer and Emotion. Abingdon: Radcliffe Medical, 1994

5 Bergevin P, Bergevin R. Recognising depression. Am J Hospice Pall Care 1995;12:22-3

6 Hinton J. The physical and mental distress of the dying. Quart J Med 1963;32:1-21

7 Fulton C. The physical and psychological symptoms experienced by patients with metastatic breast cancer before death. Eur J Cancer Care 1997;6:262-6

8 Maguire P, Howell A. Improving the psychological care of cancer patients. In: Houses A, Mayou R, Mallinson C, eds. Psychiatric Aspects of Physical Disease. London: Royal Colleges of Physicians and Psychiatrists, 1995:41-54

9 Brugha T. Depression in the terminally ill. Br J Hosp Med 1993;50: $175-81$

10 Casey P. Depression in the dying - disorder or distress? Prog Pall Care 1994;2:1-3

11 Bukberg J, Penman D, Holland J. Depression in hospitalised cancer patients. Psychosom Med 1984;46:199-211

12 Endicott J. Measurement of depression in patients with cancer. Cancer 1984;53:2243-8

13 Chochinov $\mathrm{H}$, Wilson $\mathrm{K}$, Enns $\mathrm{M}$, Lander $\mathrm{S}$. Prevalence of depression in the terminally ill: effects of diagnostic criteria and symptom threshold judgements. Am $J$ Psychiatry 1994;151: $537-40$

14 Lloyd-Williams M, Friedman T, Rudd N. A survey of psycho social service provision within hospices. Pall Med 1999;13: $431-2$

15 Faller H, Lang H, Schilling S. Emotional distress and hope in lung cancer patients, as perceived by patients, relatives, physicians, nurses and interviewers. Psycho-oncology 1995;4:21-31

16 Hoeper E, Nyez G, Kessler L, Burke J, Pierce W. The usefulness of screening for mental illness. Lancet 1984;ii:33-5

17 Zigmond AS, Snaith RP. The Hospital Anxiety and Depression Scale. Acta Psychiatr Scand 1983;67:361-70

18 Snaith P. Anhedonia: exclusion from the pleasure dome. BMJ 1992;305:134 
19 Snaith P. The concepts of mild depression. Br J Psychiatry 1987; 150:387-93

20 Ibbotson T, Maguire P, Selby T, Priestman T, Wallace L. Screening for anxiety and depression in cancer patients: the effects of disease and treatment. Eur J Cancer 1993;30A:37-40

21 Silverstone P. Is anhedonia a good measure of depression? Acta Psychiatr Scand 1991;83:249-50

22 Le Fevre P, Devereux J, Smith S, Lawrie SM, Cornbleet M. Screening for psychiatric illness in the palliative care inpatient setting: a comparison between the Hospital Anxiety and Depression Scale and the General Health Questionnaire-12. Pall Med 1999;12:399-407

23 Lloyd-Williams M, Friedman T, Rudd N. An analysis of the validity of the Hospital Anxiety and Depression scale as a screening tool in patients with advanced metastatic cancer. J Pain Symptom Management 2001;22:990-6

24 Zung W. Factors influencing the self-rating depression scale. Arch Gen Psychiatry 1967; 16:543-7

25 Dugan W, McDonald M, Passik S, Rosenfield B, Theobald D, Edgerton S. Use of Zung self-rating depression scale in cancer patients: feasibility as a screening tool. Psycho-Oncology 1998;7:483-93

26 Passik S, Lundberg J, Rosenfield B, et al. Factor analysis of the Zung self-rating depression scale in a large ambulatory oncology sample. Psychosomatics 2000;41:121-7

27 Cox J, Holden J, Sagovsky R. Detection of postnatal depression: development of 10 item Edinburgh Postnatal Depression Scale. Br J Psychiatry 1987; 150:782-6

28 Cox J, Chapman G, Murray D, Jones P. Validation of the Edinburgh postnatal scale (EPDS) in non-postnatal women. J Affect Disord 1996;39:185-9

29 Lloyd-Williams M, Friedman T, Rudd N. Criterion validation of the Edinburgh Postnatal Depression Scale as a screening tool for depression in patients with advanced metastatic cancer. $J$ Pain Symptom Management 2000;20:259-65

30 Chochinov H, Wilson K, Enns M, Lander S. Are you depressed? Screening for depression in the terminally ill. Am J Psychiatry 1997; 154:674-6

31 Mahoney J, Drinka T, Abler R, et al. Screening for depression: single question versus GDS. J Am Geriatr Soc 1994;42:1006-8

32 Lloyd-Williams M, Taylor F, Dennis M. Is asking UK palliative care patients the single question 'are you depressed?' a robust method of screening for depression? BMJ 2003;327:372-3

33 Kovacs M, Beck A, Weissman A. Hopelessness; an indicator of suicidal risk. Suicide 1975;5:98-103

34 Brown J, Henteleff P, Barakat S, Rowe C. Is it normal for terminally ill patients to desire death? Am J Psychiatry 1986;143:208-11

35 Chochinov H, Wilson K, Enns M, et al. Desire for death in the terminally ill. Am J Psychiatry 1995;152:1185-91

36 Tiernan E, Casey P, O'Boyle C, et al. Relations between desire for early death, depressive symptoms and antidepressants prescribing in terminally ill patients with cancer. $J$ R Soc Med 2002;95:386-90

37 Lloyd-Williams M. How common are thoughts of self-harm in a UK palliative care population? Supportive Care Cancer 2002;10:422-4

38 Lloyd-Williams M, Friedman T, Rudd N. A survey of antidepressant prescribing in the terminally ill. Pall Med 1999;13:243-8
39 Farebarrow N, Gamzler S, Cutter F, Reynolds D. An eight year survey of hospital suicides. Life-threatening Behaviour 1971;1:184-202

40 Bolund C. Suicide and cancer 1. Demographic and social characteristics of cancer patients who committed suicide in Sweden, 1973-1976. J Psychosoc Oncol 1985;3:17-30

41 Bolund C. Suicide and cancer 11, medical and care factors in suicides by cancer patients in Sweden, 1973-1976. J Psychosoc Oncol 1985;3:31-52

42 Linn M, Linn B, Harris R. Effects of counselling for late stage cancer patients. Cancer 1982;49:1048-55

43 Spiegel D. Cancer and depression. Br J Psychiatry 1996;168:109-16

44 Parle M, Jones B, Maguire P. Maladaptive coping and affective disorders among cancer patients. Psychol Med 1996;26:735-44

45 Greer S, Moorey S. Adjuvant psychological therapy for cancer patients. Pall Med 1997;11:240-4

46 Moorey S, Greer S, Bliss J, Law M. A comparison of adjuvant psychological therapy and supportive counselling in patients with cancer. Psycho-Oncology 1998;7:218-28

47 Chilvers C, Dewey M, Fielding K, et al. Antidepressant drugs and generic counselling for treatment of major depression in primary care: randomised trial with patient preference arms. BMJ 2001;322:772-5

48 Maguire P. The use of antidepressants in patients with advanced cancer. Supportive Care Cancer 2000;8:265-7

49 Chaturvedi S, Maguire P, Hopwood P. Antidepressant medication in cancer patients. Psycho-Oncology 1994;3:57-60

50 Sindrup SH, Gram LF, Brosen K, Eshoj O, Mogensen EF. The selective serotonin reuptake inhibitor paroxetine is effective in the treatment of diabetic neuropathy symptoms. Pain 1990;42:135-44

51 Goodnick PJ, Jimenez I, Kumar A. Sertraline in diabetic neuropathy: preliminary-results. Ann Clin Psychiatr 1997;9:255-7

52 Razavi D, Allilaire J, Smith M, et al. The effect of fluoxetine on anxiety and depression symptoms in cancer patients. Acta Psychiatr Scand 1996;94:205-10

53 Goodnick P, Hernandez M. Treatment of depression in co-morbid medical illness. Expert Opin Pharmacother 2000;7:1367-84

54 Martin R, Hilton S, Derry S, Richards N. General practitioners' perceptions of the tolerability of antidepressant drugs: a comparison of selective serotonin inhibitors and tricyclic antidepressants. BMJ 1997;314:646-51

55 Pereira J, Bruera E. Depression with psychomotor retardation: diagnostic challenges and the use of psychostimulants. J Pall Med 2001;4:15-21

56 Rozans M, Dreisbach A, Lertora J, Kahn M. Palliative use of methylphenidate in patients with cancer: a review. J Clin Oncol 2002;20:335-69

57 Dein S, George R. The use of psychostimulants by palliative care consultants in the UK: a retrospective telephone survey. Pall Med 2002;16:167-8

58 Payne S. Depression in palliative care patients: a literature review. Int J Pall Nurs 1998;4:184-91

59 Stiefel F, Die-Trill M, Berney A, Loarte J, Razavi A. Depression in palliative care: a pragmatic report from the expert working party of the European Association for Palliative Care. Supportive Care Cancer 2001;9:477-88 